ORIGINAL ARTICLE

\title{
Physical Activity and Life Quality in Patients with Asthma and Osteoarthritis
}

YU. S. IVANCHUK ${ }^{1}$, L. V. TRIBUNTCEVA ${ }^{1}$, A. V. BUDNEVSKY ${ }^{1}$, N. I. OSTROUSHKO ${ }^{2}$, YA. S. SHKATOVA $^{1}$, R. E. $^{2}$ TOKMACHEV ${ }^{1}$, I. A. SAVUSHKINA ${ }^{1}$

1 Voronezh State Medical University

${ }^{2}$ Health department of Voronezh region

Yuliya Sergeevna Ivanchuk - post graduate student at the department of Faculty Therapy, Voronezh State Medical University, 394036, Voronezh, Russia. Ludmila Vasil'evna Tribuntceva - PhD, associate professor at the department of general practice, Voronezh State Medical University, 394036, Voronezh, Russia Andrey Valerievich Budnevsky - M.D., PhD, DSc, prof., head of the department of Faculty Therapy, Voronezh State Medical University, 394036, Voronezh, Russia.

Nadezhda Igorevna Ostroushko - head of the department of medical assistance to the adult population at the Health department of Voronezh region Yanina Sergeevna Shkatova - postgraduate student at the department of Faculty Therapy, Voronezh State Medical University, 394036, Voronezh, Russia. Roman Evgenievich Tokmachev - PhD, associate professor at the department of Faculty Therapy, Voronezh State Medical University, 394036, Voronezh, Russia.

Inessa Alekseevna Savushkina - 2d year resident at the department of Faculty Therapy, Voronezh State Medical University, 394036, Voronezh, Russia. Corresponding author: Yanina Sergeevna Shkatova: Moskovsky prospect 60-32, Voronezh, Russia, 394016. Phone number: +7-961-187-58-00. Email: yankashkat@gmail.com

\begin{abstract}
Background. Physical activity is associated with better asthma control and life quality in asthma. Osteoarthritis is one of the less studied comorbidities in asthmatic patients.

Methods. The study included 38 patients diagnosed with asthma, 65 patients with asthma and osteoarthritis, and 40 volunteers who did not suffer from asthma and osteoarthritis. During the study, 3 groups were formed: Group 1 consisted of patients with asthma; Group 2 included patients with both asthma and osteoarthritis, Control group consisted of volunteers. Spirometry, Asthma Quality of Life Questionnaire (AQLQ), Asthma Control Test (ACT) were used in asthmatic patients. International Physical Activity Questionnaire (IPAQ) in its short from was filled by all the participants.

Results. Both Group 1 and Group 2 did not engage in vigorous physical activity. Median of MET-min/week total (1825) was significantly less in Group 2 compared with Control and Group 1 ( $p=0.0000$ and $p=0.0169$, respectively). MET-min/week total had positive correlations with ACT ( $r=0.50, p<0.05), A Q L Q(S)$ total $(r=0.58$, $p<0.05)$, AQLQ(S) activity domain $(r=0.28, p<0.05), A Q L Q(S)$ emotions domain $(r=0.24, p<0.05), A Q L Q(S)$ symptoms domain $(r=0.34, p<0.05)$, FVC $(r=0,28, p<0.05)$, FEV1 $(r=0,37, p<0.05)$, Index Tiffno $(r=0,18, p<0.05)$. Minutes/week sitting time had a negative correlation with ACT values $(r=-0.33, p<0.05), A Q L(S)$ total values ( $r=-$ $0.39, \mathrm{p}<0.05)$.

Conclusion. Patients with asthma and osteoarthritis spend significantly less time on moderate activity, walking compared with asthmatics not suffering from osteoarthritis; they avoid vigorous activity. Higher physical activity is associated with better life quality, asthma control and lung function, thus paying attention to osteoarthritis in asthmatic patients is crucial.
\end{abstract}

Key words: life quality, physical activity, asthma, osteoarthritis

\section{INTRODUCTION}

Bronchial asthma (BA) is a heterogeneous disease characterized by chronic inflammation of the airways, the presence of respiratory symptoms such as wheezing, shortness of breath and cough that vary in time and intensity [1-3]. According to World Health Organization in 2016 up to 339 million people of all ages worldwide suffered from the disease. BA is proven to be one of the most common causes of morbidity and mortality worldwide; it is a major source of the global economic burden when it comes to both direct and indirect costs [4]. Nowadays, the goal of asthma treatment is to have full control over symptoms of the disease so that patients can have normal levels of activity and achieve a good quality of life, which is why the Global Strategy for Asthma Management and Prevention (GINA) includes recommendations on regular physical activity for asthmatics that will allow them to improve their overall health. Several studies report that higher physical activity is associated with better asthma control, lung function parameters and also a decreased number of exacerbations and doctor's appointments $[5,6]$. However, some studies also show that patients with asthma spend less time being physically active and/or have less intense physical activities compared with those who do not suffer from BA. According to a study by Teramoto M. et al., which included 3840 people, over $30 \%$ of asthmatics did not have leisure-time physical activity compared with about $23 \%$ of those who did not suffer from asthma [7]. The time that asthmatic patients spend on doing physical activities is also significantly less compared with those without asthma. Yawn B.P. et al. showed that over 33\% of adult women did not have the recommended 15 minutes or more of moderate activity for at least 5 days per week; poor asthma control was also associated with low physical activity in the study [8].

Osteoarthritis (OA) is one of the less studied comorbidities of asthma. It has been shown that prevalence of osteoarthritis in patients with asthma is significantly higher compared with chronic obstructive pulmonary disease and subjects with no respiratory diseases $[9,10]$. Mahmood Z. et al. found that asthmatic patients who had higher rate of emergency hospitalizations also had a higher risk of developing OA [11]. Physical activity is also very important for patients with osteoarthritis, it reduces pain 
and is associated with better physical function and quality of life [12]. To our knowledge there were no previous studies assessing the effect of osteoarthritis on physical activity and quality of life in asthmatic patients.

Objective: to assess physical activity and life quality in asthmatic patients compared with patients who suffer from both asthma and osteoarthritis.

\section{MATERIALS AND METHODS}

Patients were included in the study according to the following criteria: age from 30 to 70 years, mild to moderate asthma or mild to moderate asthma with a diagnosed osteoarthritis, patients' informed consent for participation in the study. Volunteers, who did not suffer from asthma and osteoarthritis were also included in the study. Patients were excluded, according to the following criteria: mental illness, tuberculosis of any localization, severe and decompensated diseases of liver and kidneys, severe and decompensated cardiovascular diseases (acute period of myocardial infarction, unstable angina, transient ischemic attack, intracerebral hemorrhage, acute heart failure, etc.), cancer; pregnancy and lactation, severe infectious diseases (HIV infection, syphilis, progressive course of viral hepatitis $B$ and $\mathrm{C}$ ), history of any rheumatic disease, fibromyalgia and other chronic painful condition, knee/hip surgery, traumatic osteoarthritis.

All patients gave their informed consent for inclusion before they participated in the study. The study was conducted in accordance with the Declaration of Helsinki, and the protocol was approved by the Ethics Committee of Voronezh State Medical University (protocol no. 1 from February 21, 2018).

The diagnosis of asthma was made according to GINA; complaints, anamnesis data, objective status data, laboratory and instrumental data (spirometry with a 400-mg salbutamol test) were analyzed. All patients received standard asthma therapy. Patients with asthma and osteoarthritis filled in Asthma Quality of Life Questionnaire (AQLQ), Asthma Control Test (ACT) and International Physical Activity Questionnaire (IPAQ) in its short from. Volunteers who did not suffer from asthma and osteoarthritis only filled in short from of IPAQ.

Statistical data processing was performed using the STATGRAPHICS 5.1 Plus for Windows software package. Quantitative data at normal distribution are presented in the form $M \pm \sigma$, where $M$ is a mean, $\sigma$ is a standard deviation. Qualitative variables were compared using the $x 2$ test. Comparison of quantitative parameters was performed using one-way analysis of variance (ANOVA). Differences were considered statistically significant at a level of $p$ values $<0.05$. The data that is not normally distributed is presented as median values and interquartile ranges. Nonnormally distributed data was assessed using KruskalWallis test, the differences were considered significant at a level of $p$ values $<0.05$.

\section{RESULTS}

In total, the study included 103 patients (there were 34 men (33.01\%) and 69 women (66.99\%)) diagnosed with mild to moderate asthma, whose average age was $58.52 \pm 7.14$ years. Aside from patients volunteers who did not suffer from asthma and/or osteoarthritis were also included - 40 people (13 men (32.5\%) and 27 women (67.5\%)) whose average age was $56.41 \pm 8.12$ years.

Three groups were formed - Group 1 which included patients with asthma and without osteoarthritis; Group 2 patients with both asthma and osteoarthritis; and Control group - volunteers who did not suffer from BA and/or OA. The group with asthma included 38 patients and the group with asthma and osteoarthritis consisted of 65 patients. The formed groups did not differ by age, gender, marriage status and level of education $(p<0.05)$.

The results on vigorous and moderate activity in the studied groups are presented in Table 1, total activity, walking and sitting time - in Table 2.

According to IPAQ there was a significant difference in vigorous activity between Control group and Groups 1 and 2: volunteers from Control group had more days of vigorous activity per week $(p=0.0000 ; p=0.0000$, respectively), more minutes of vigorous activity per week $(p=0.0000 ; p=0.0005$, respectively), and more MET$\mathrm{min} /$ week of vigorous activity $(p=0.0000 ; p=0.0000$, respectively). There was no significant difference in vigorous activity between Group 1 and Group 2: in both groups parameters of vigorous activity were very low $(p>0.05)$. There was no significant difference in the number of days that volunteers from Control group spent on moderate activity per week compared with Group 1 $(p=0.4605)$ and group $2(p=0.1256)$, however, Control subjects spent more time and energy on moderate activity compared with both Group 1 and 2. Patients from Group 1 spent more days/week $(p=0.1032)$, more minutes/week $(p=0.0239)$, more MET-minutes/week $(p=0.0239)$ on moderate physical activity compared with Group 2. Subjects from Control group had higher number of days they spent walking during the week compared with Group 2 $(p=0.0169)$, but not Group $1(p=0.4605)$. Control group also had higher values of such parameters of IPAQ as minutes/week walking, MET-min/week+walking and MET$\mathrm{min} /$ week total compared with both Group 1 and $2(\mathrm{p}<0.05)$. On contrary, volunteers from Control group spent less time sitting than patients from $2(p=0.0000)$, however, there was not a significant difference with Group $1 \quad(p=0.9131)$. Patients from Group 1 spent more days on walking during the week $(p=0.0139)$, they also spent more time on it $(p=0.0045)$, the amount of MET-min/week spent on walking was also significantly higher $(p=0.0045)$ compared with Group 2. Patients from Group 2 had more minutes/week of sitting time than patients from Group 1 and the difference was significant $(p=0.0217)$. Total amount of MET-min/week was significantly higher in Group 1 compared with Group 2 $(p=0.0169)$.

There was no significant difference in spirometry parameters between Group 1 and Group 2 (Table 3).

$A Q L Q(S)$ activity domain was decreased in patients with asthma and osteoarthritis compared with asthmatic patients: $3.46 \pm 0.56$ points and - $4.70 \pm 1.08$ points, respectively $(p=0.0000)$. AQLQ(S) emotions domain was also decreased in patients with asthma and osteoarthritis $3.50 \pm 0.61$ points compared with $4.68 \pm 1.10$ points in patients from Group $1(p=0.0000)$. AQLQ(S) environment domain did not differ significantly $(p=0.1635)$. AQLQ(S) symptoms domain was $4.38 \pm 0.85$ points and $-3.34 \pm 0.53$ points in Group 1 and Group 2, respectively $(p=0.0000)$. 
AQLQ(S) general value was significantly higher in asthmatic patients without osteoarthritis $(4.69 \pm 1.11$ points vs $3.49 \pm 0.59$ points in asthmatics with osteoarthritis $(p=0.0000))$.

Values of ACT test were $18.0 \pm 2.61$ points and $16.78 \pm 1.92$ points in the Group 1 and Group 2, respectively ( $p=0.0079)$.

MET-min/week total had positive correlations with ACT $(r=0.50, p<0.05), A Q L Q(S)$ total $(r=0.58, p<0.05)$,
$A Q L Q(S)$ activity domain $(r=0.28, \quad p<0.05), \quad A Q L Q(S)$ emotions domain $(r=0.24, p<0.05), A Q L Q(S)$ symptoms domain $(r=0.34, p<0.05)$, FVC $(r=0.28, p<0.05)$, FEV1 $(r=0.37, p<0.05)$, Index Tiffno $(r=0.18, p<0.05)$.

Minutes/week sitting time had a negative correlation with ACT values $(r=-0.33, p<0.05), A Q L Q(S)$ total values $(r=-0.39, p<0.05)$.

Table 1. Vigorous and moderate activity according to IPAQ

\begin{tabular}{|c|c|c|c|c|}
\hline & Control & Group 1 & Group 2 & $p$-value \\
\hline Days/week VPA & $3[5 ; 2]$ & $0[1,75 ; 0]^{*}$ & $0[2 ; 0]^{\star \star}$ & $\begin{array}{l}p=0,0000^{*} \\
p=0,9783 \\
p=0,0000^{\star *}\end{array}$ \\
\hline Minutes/week VPA & $120[242,5 ; 120]$ & $0[30 ; 0]^{*}$ & $0[30 ; 0]^{\star *}$ & $\begin{array}{l}p=0,0005^{\star} \\
p=0,9291 \\
p=0,0000^{\star *}\end{array}$ \\
\hline MET-min/week VPA & 960 [1940; 480] & $0[240 ; 0]^{*}$ & $0[240 ; 0]^{\star *}$ & $\begin{array}{l}p=0,0000^{*} \\
p=0,9291 \\
p=0,0000^{* *}\end{array}$ \\
\hline Days/week MPA & $5[6,25 ; 4]$ & $5[6,75 ; 5]$ & $5[7 ; 2]$ & $\begin{array}{l}\mathrm{p}=0,4605 \\
\mathrm{p}=0,1032 \\
\mathrm{p}=0,1256\end{array}$ \\
\hline Minutes/week MPA & $450[545 ; 450]$ & $210[350 ; 150]^{*}$ & $140[250 ; 150]^{\# \text { **}}$ & $\begin{array}{l}p=0,0005^{\star} \\
p=0,0239^{\#} \\
p=0,0000^{\star *}\end{array}$ \\
\hline MET-min/week MPA & $1800[2180 ; 920]$ & $840[1400 ; 600]^{*}$ & $560[1000 ; 0]^{\# * *}$ & $\begin{array}{l}p=0,0005^{*} \\
p=0,0239^{\#} \\
p=0,0000^{* *}\end{array}$ \\
\hline
\end{tabular}

Footnote: data are presented as median values and interquartile ranges; *the differences are significant at $p<0.05$ between Control group and Group 1, \# the differences are significant at $p<0.05$ between Group 1 and Group 2; ${ }^{* *}$ the differences are significant at $p<0.05$ between Control group and Group 2; VPA - vigorous physical activity, MPA - moderate physical activity, MET - metabolic equivalent of task

Table 2. Total activity and sitting time according to IPAQ

\begin{tabular}{|c|c|c|c|c|}
\hline (2) & Control & Group 1 & Group 2 & $p$-value \\
\hline Days/week walking & $7[7 ; 6,75]$ & $7[7 ; 7]$ & $7[7 ; 5]^{\# \star \star}$ & $\begin{array}{l}p=0,9681 \\
p=0,0139^{\#} \\
p=0,0169^{* *}\end{array}$ \\
\hline Minutes/week walking & $420[577,5 ; 300]$ & $350[402,5 ; 202,5]^{*}$ & $210[350 ; 120]^{\# \star *}$ & $\begin{array}{l}p=0,0392^{*} \\
p=0,0045^{\#} \\
p=0,0000^{* *}\end{array}$ \\
\hline Days/week MPA + walking & $12[13 ; 10,75]$ & $12[13,75 ; 10]$ & $10[12 ; 7]^{\#}$ & $\begin{array}{l}p=0,7172 \\
p=0,0045^{\#} \\
p=0,0056^{\star *}\end{array}$ \\
\hline Minutes/week MPA + walking & $780[955 ; 650]$ & $580[700 ; 335]^{*}$ & $390[630 ; 180]^{\# \star *}$ & $\begin{array}{l}p=0,0003^{*} \\
p=0,0118^{\#} \\
p=0,0000^{* *}\end{array}$ \\
\hline Minutes/week MVPA & $495[695,2 ; 420]$ & $250[350 ; 150]^{*}$ & $180[320 ; 60]^{\# * *}$ & $\begin{array}{l}p=0,0000^{*} \\
p=0,0435^{\#} \\
p=0,0000^{* *}\end{array}$ \\
\hline $\begin{array}{l}\text { Minutes/week MVPA + } \\
\text { walking }\end{array}$ & $\begin{array}{l}930[1087,5 ; \\
777,5]\end{array}$ & $620[715 ; 335]^{\star}$ & $500[670 ; 210]^{\# \star \star}$ & $\begin{array}{l}p=0,0000^{*} \\
p=0,0153^{\#} \\
p=0,0000^{* *}\end{array}$ \\
\hline MET-min/week walking & $\begin{array}{l}1386[1905,75 ; \\
990]\end{array}$ & $1155[1328,25 ; 668,25]^{*}$ & $\begin{array}{l}693[1155 \\
396]^{\# \star \star}\end{array}$ & $\begin{array}{l}p=0,0392^{*} \\
p=0,0045^{\#} \\
p=0,0000^{* *}\end{array}$ \\
\hline MET-min/week total & $\begin{array}{l}3829,5[4539,75 ; \\
3241,25]\end{array}$ & $2535[2895,75 ; 1228,5]^{*}$ & $1825[2408 ; 756]^{\# \star \star}$ & $\begin{array}{l}p=0,0000^{*} \\
p=0,0169^{\#} \\
p=0,0000^{\star *}\end{array}$ \\
\hline Minutes/week sitting time & $240[300 ; 120]$ & $300[300 ; 240]$ & $360[420 ; 240]^{\# \star *}$ & $\begin{array}{l}p=0,9131^{*} \\
p=0,0217^{\#} \\
p=0,0000^{\star *}\end{array}$ \\
\hline
\end{tabular}

Footnote: data are presented as median values and interquartile ranges; *the differences are significant at $p<0.05$ between Control group and Group 1, \# the differences are significant at $p<0.05$ between Group 1 and Group 2; ${ }^{\star *}$ the differences are 
significant at $p<0.05$ between Control group and Group 2; MPA - moderate physical activity, MVPA - moderate plus vigorous activity, MET - metabolic equivalent of task

Table 3. Lung function in the studied groups

\begin{tabular}{|l|l|l|l|}
\hline Parameter & Group 1 & Group 2 & $\mathrm{p}$-value \\
\hline FVC, \% & $91,6[102,1 ; 87]$ & $93[102,1 ; 88,5]$ & $\mathrm{p}=0,9324$ \\
\hline FEV1, \% & $65,65[84,12 ; 62]$ & $68,7[80,33 ; 66,06]$ & $\mathrm{p}=0,8921$ \\
\hline Index Tiffno, \% & $74,84[82,81 ; 70,6]$ & $73,8[82,3 ; 70]$ & $\mathrm{p}=0,8724$ \\
\hline
\end{tabular}

Footnote: data are presented as median values and interquartile ranges; *the differences are significant at $p<0.05$

\section{DISCUSSION}

The beneficial effects of physical activity on health and life expectancy have been shown in a number of studies. It is believed that physically active people live on average 3-4 years longer, and changes in health and performance are often the result of not aging, but lack of physical activity [13]. Moderate aerobic physical activity (at least 150 minutes per week) reduces the risk of coronary heart disease, arterial hypertension, diabetes mellitus and depression; physical activity of 150 to 300 minutes per week reduces the risk of becoming overweight, colon cancer and mammary tumors [14-16]. Even a little physical activity is better than no physical activity at all; it is enough to gradually increase the duration of exercising until the required minimum level of physical activity is achieved. Physical exercise is recommended for the elderly (over 65 years of age), as well as for those who suffer from chronic diseases. However, asthmatic patients are often afraid to engage in physical activity. Analysis of psychological barriers that interfered with active lifestyles in patients with asthma showed that a high level of psychological barriers was significantly correlated with increased asthma symptoms, anxiety and depression, low quality of life and amount of oral glucocorticosteroids used in the last 12 months [17]. Asthmatic patients are especially avoidant when it comes to a vigorous activity as it is associated with exercise-induced bronchoconstriction [18]. A study by Verlaet $A$. et al. showed that there was an association between uncontrolled asthma and vigorous physical activity but only in women. Men tended to have more vigorous physical activity if they had well controlled asthma [19]. It was also reported that patients with severe asthma had less physical activity compared with other asthmatics [20]. In our study patients suffered from mild to moderate asthma, however, they did not engage in vigorous activity. Patients with both asthma and OA also did not engage in vigorous physical activity too. Similar results are described in the study by Farr J.N. et al., where physical activity in patients with knee osteoarthritis was assessed with use of accelerometer [21]. In our study patients with asthma spent less time on moderate physical activity than controls and more than asthmatics with OA. That is in accordance with the scientific data that moderate physical activity carries less risk of asthma attack compared with high intensity physical activity [22]. Patients with OA also spend more time on moderate physical activity than on vigorous one [21]. While excessive physical activity is found to be a risk factor for OA, moderate physical activity was reported to have beneficial effects in knee, hip and even hand $O A$, as it decreases pain, improves physical function and mobility of joints [23].
IPAQ allows to distinguish walking from other types of physical activities: in our study asthmatic patients spent less time walking compared with controls and more time walking than asthmatic patients with OA. This could be explained by the results of the study by King LK et al [24]. Authors included 18490 participants in their study, and they have found that walking difficulty was associated with hip/knee $O A$ and its probability increased with a higher number of joints involved in the pathological process. However, daily walking reduces functional limitations in knee OA rather than causing worsening of the condition; it is recommended to walk $\geq 6000$ steps/day [25].

Total weekly activity according to IPAQ was significantly higher in control group compared with asthmatics and patients with both asthma and OA. IPAQ total activity score was also significantly higher in patients with asthma compared with asthmatics also suffering from $\mathrm{OA}$. According to IPAQ, total physical activity of at least 600 MET-minutes/week is considered to be moderate physical activity. In our study all groups reached the level of moderate physical activity which is in accordance with other studies that relied on IPAQ for evaluating physical activity levels in patients with asthma and OA. In a study by Gay C. et al. it was shown that the median IPAQ total activity score was $2628 \mathrm{MET}$-min/week in patients with OA [26]. In a study by Verlaet $A$ et al, the median IPAQ total activity score was $2000 \mathrm{MET}$-min/week in male asthmatics with controlled asthma, $2253 \mathrm{MET}-\mathrm{min} /$ week in uncontrolled, $1732.50 \mathrm{MET}$-min/week in female asthmatics with controlled asthma, $2273 \mathrm{MET}$-min/week in uncontrolled female asthmatics [19]. Our study showed similar numbers in asthmatic patients (median of 2535 MET-min/week), and the number was lower in asthmatics with OA (median of $1825 \mathrm{MET}$-min/week), suggesting worsening effect these conditions have on each other.

Not many studies concentrated on sitting time in asthmatic patients, available information suggests no significant difference in sitting time between asthmatics and controls not suffering from asthma [19]. Accordingly, our study shows that there is no significant difference between control group and group of asthmatic patients, however, the difference is significant between asthmatics and patients with $B A$ and $O A$, which could be explained by the fact that patients with BA and OA are also limited by joints pain, having to face walking difficulty (spending less time on it) and thus spending more time on sitting activities.

Our study also showed associations between higher physical activity and better quality of life (according to $A Q L Q(S)$ ), better asthma control and better lung function which is in agreement with the scientific data available at this point. However, there are small differences. Metaanalysis by $\mathrm{Wu} \mathrm{X}$ et al. reports that regular continuous 
aerobic exercise leads to improvement of FEV1, PEF, FVC,

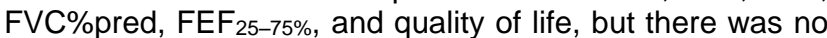
improvement noted in FEV1\%pred and FEV1/FVC\% [26]. Another study by Brumpton BM et al, which included 1329 patients, showed that physical activity slowed lung function decline, especially $\mathrm{FEV}_{1}, \mathrm{FEV}_{1} / \mathrm{FVC}$ ratio and PEF [27].

\section{CONCLUSION}

Our study showed that asthmatic patients spend less time on physical activity compared with volunteers who do not suffer from asthma. Asthmatic patients tend to avoid vigorous activity and prefer moderate physical activity and walking. To our current knowledge there are no other studies investigating physical activity in patients with both osteoarthritis and bronchial asthma. Such patients spend significantly less time on moderate activity, walking compared with asthmatics not suffering from osteoarthritis; they also avoid vigorous activity. Osteoarthritis is a serious but often underestimated comorbidity of asthma which influences physical activity in asthmatic patients. Higher physical activity is associated with better life quality, asthma control and lung function, which is why paying attention to osteoarthritis, treating it, and explaining the necessity of physical activity to patients suffering from both asthma and osteoarthritis is especially important.

\section{REFERENCES}

1. Global Strategy for Asthma Management and Prevention. Updated 2020. Available at: https://ginasthma.org/wpcontent/uploads/2020/06/GINA-2020-report_20_06_04-1wms.pdf

2. Budnevsky A, Tribuntceva L, Kozhevnikova S, Ovsyannikov E. Impact of Metabolic Syndrome Components on Asthma Control and Life Quality of Patients. International Journal of Biomedicine. 2018;8(1):33-6. doi: 10.21103/article8(1)_oa4.

3. Budnevsky AV, Malysh EY, Ovsyannikov ES, Drobysheva ES. Asthma and metabolic syndrome: Clinical and pathogenetic relationships. Ter Arkh. 2015;87(10):110. Russian. doi: 10.17116/terarkh20158710110-114.

4. Ermolova AV, Budnevsky AV, Malysh E Yu, Ovsyannikov ES, Drobysheva ES. Bronchial asthma and metabolic syndrome. Klin Med (Mosk). 2015;93(6):44-9. Russian.

5. Wu X, Gao S, Lian Y. Effects of continuous aerobic exercise on lung function and quality of life with asthma: a systematic review and meta-analysis. J Thorac Dis. 2020;12(9):47814795. doi:10.21037/jtd-19-2813.

6. Provotorov VM, Budnevsky AV, Filatova YI, Perfil'eva MV. Antioxidant therapy of bronchial asthma. Klin Med (Mosk). 2015;93(8):19-22. Russian.

7. Teramoto M, Moonie S. Physical activity participation among adult Nevadans with self-reported asthma. J Asthma. 2011;48(5):517-522. doi:10.3109/02770903.2011.567426.

8. Yawn BP, Rank MA, Bertram SL, Wollan PC. Obesity, low levels of physical activity and smoking present opportunities for primary care asthma interventions: an analysis of baseline data from The Asthma Tools Study. NPJ Prim Care Respir Med. 2015;25:15058. doi:10.1038/npjpcrm.2015.58.

9. Koo HK, Song $\mathrm{P}$, Lee JH. Novel association between asthma and osteoarthritis: a nationwide health and nutrition examination survey. BMC Pulm Med. 2021;21(1):59. doi:10.1186/s12890-021-01425-6.

10. Budnevsky AV, Ovsyannikov ES, Labzhania NB. Chronic obstructive pulmonary disease concurrent with metabolic syndrome: Pathophysiological and clinical features. Ter Arkh. 2017;89(1):123-127. Russian. doi: 10.17116/terarkh2017891123-127.
11. Zainab A Mahmood, Ziyad T Malghooth. Relationship Of Hips And Knees Osteoarthritis With Bronchial Asthma. Res J Pharm Biol Chem Sci. 2019; 10(2): 64-70.

12. Lin W, Alizai H, Joseph GB, Srikhum W, Nevitt MC, Lynch $\mathrm{JA}$, et al. Physical activity in relation to knee cartilage T2 progression measured with $3 \mathrm{~T} \mathrm{MRI}$ over a period of 4 years: data from the Osteoarthritis Initiative. Osteoarthritis Cartilage. 2013;21(10):1558-66. doi: 10.1016/j.joca.2013.06.022.

13. Langhammer B, Bergland A, Rydwik E. The Importance of Physical Activity Exercise among Older People. Biomed Res Int. 2018;2018:7856823. doi:10.1155/2018/7856823.

14. Brown JC, Winters-Stone K, Lee A, Schmitz KH. Cancer, physical activity, and exercise. Compr Physiol. 2012;2(4):2775-2809. doi:10.1002/cphy.c120005.

15. Winzer EB, Woitek F, Linke A. Physical Activity in the Prevention and Treatment of Coronary Artery Disease. J Am Heart Assoc. 2018;7(4):e007725. doi:10.1161/JAHA.117.007725.

16. Shaphe MA, Chahal A. Relation of Physical Activity with the Depression: A Short Review. J Lifestyle Med. 2020;10(1):16. doi:10.15280/jlm.2020.10.1.1.

17. Freeman AT, Hill D, Newell C, Moyses H, Azim A, Knight D, et al. Patient perceived barriers to exercise and their clinical associations in difficult asthma. Asthma Res Pract. 2020;6:5. doi: 10.1186/s40733-020-00058-6.

18. Aggarwal B, Mulgirigama A, Berend N. Exercise-induced bronchoconstriction: prevalence, pathophysiology, patient impact, diagnosis and management. NPJ Prim Care Respir Med. 2018;28(1):31. doi:10.1038/s41533-018-0098-2.

19. Verlaet A, Moreira A, Sá-Sousa A, Barros R, Santos R, Moreira $\mathrm{P}$, et al. Physical activity in adults with controlled and uncontrolled asthma as compared to healthy adults: a cross-sectional study. Clin Transl Allergy. 2013;3(1):1. doi: 10.1186/2045-7022-3-1.

20. Hennegrave F, Le Rouzic O, Fry S, Behal H, Chenivesse C, Wallaert B. Factors associated with daily life physical activity in patients with asthma. Health Sci Rep. 2018;1(10):e84. doi:10.1002/hsr2.84.

21. Farr JN, Going SB, Lohman TG, Rankin L, Kasle S, Cornett $M$, et al. Physical activity levels in patients with early knee osteoarthritis measured by accelerometry. Arthritis Rheum. 2008:59(9):1229-36. doi: 10.1002/art.24007.

22. Del Giacco SR, Garcia-Larsen V. Aerobic exercise training reduces bronchial hyper-responsiveness and serum proinflammatory cytokines in patients with asthma. Evid Based Med. 2016;21(2):70. doi: 10.1136/ebmed-2015-110260.

23. Rausch Osthoff AK, Niedermann K, Braun J, Adams J, Brodin N, Dagfinrud H, et al. 2018 EULAR recommendations for physical activity in people with inflammatory arthritis and osteoarthritis. Ann Rheum Dis. 2018;77(9):1251-1260. doi: 10.1136/annrheumdis-2018-213585.

24. King LK, Kendzerska T, Waugh EJ, Hawker GA. Impact of Osteoarthritis on Difficulty Walking: A Population-Based Study. Arthritis Care Res (Hoboken). 2018;70(1):71-79. doi: 10.1002/acr.23250.

25. White DK, Tudor-Locke C, Zhang Y, Fielding R, LaValley M, Felson DT, et al. Daily walking and the risk of incident functional limitation in knee osteoarthritis: an observational study. Arthritis Care Res (Hoboken). 2014;66(9):1328-36. doi: 10.1002/acr.22362.

26. Wu X, Gao S, Lian Y. Effects of continuous aerobic exercise on lung function and quality of life with asthma: a systematic review and meta-analysis. J Thorac Dis. 2020;12(9):47814795. doi:10.21037/jtd-19-2813.

27. Brumpton BM, Langhammer $A$, Henriksen $A H$, Camargo $C A$ Jr, Chen Y, Romundstad PR, et al. Physical activity and lung function decline in adults with asthma: The HUNT Study. Respirology. 2017;22(2):278-283. doi: 10.1111/resp.12884. 